\title{
Editorial
}

\section{Approaches and Impact}

Sarah Morse

Northumbria University, UK

sarah.morse@northumbria.ac.uk

With the start of the new academic year, many of us will have hoped for a return to some 'normality' in terms of our teaching and ways of working. Unfortunately, however, in light of the continuing pandemic, this is likely to look different for some further time to come and whilst some will be experiencing a move back to predominantly face to face teaching, others might be taking a more blended approach or seeing a continuation of online work. Regardless, and despite the many challenges, it is evident that invaluable and innovative teaching and research in the field of PLE has continued over the last 18 months. I anticipate that others, like myself, might be considering how what we have learnt over this period can potentially benefit or translate to our teaching and research this year and beyond. With that in mind I hope that this issue of the journal provides food for thought and encourages you to continue to share details of your work- something which is so important for development in the field.

Our first article is a fascinating exploration of the relationship between legal capability and the capabilities approach. The concept of legal capability (with its focus on 
possessing knowledge, skills and attitudes to recognise, deal with and resolve law related issues) is central to our understanding of PLE. Professor Dawn Watkins reflects upon the commonly held view that this is closely linked to the capabilities approach (which, in broad terms, is stated to evaluate human wellbeing with reference to individual freedoms rather than in monetary or material terms) and examines the history of this, the true nature of the relationship and, in concluding that the relationship is in fact more disparate, considers the pros and cons of 'reimagining' the relationship to bring these two fields closer together. There is much for the reader to ponder here including the desirability of this, with potential scope for further discussion and exploration.

Our second article provides an evaluation of the impact of Street Law programmes on the law student instructors. Brandon Golob explores whether participation impacts upon students' mastery and performance orientated goals as well as providing evidence of the impact on law students self-efficacy for communicating legal information to non-lawyers, public speaking, developing lawyer/client relationships and soft skills (such as empathy). Evaluation relating to the impact of PLE is of interest and importance to us all- potentially providing an evidence base upon which to sustain and develop our programmes and work -so this article is a welcome addition to that. 
Further discussion regarding the benefit of PLE programmes for law students is found in our third article. Here, Keren Lloyd Bright, with Maria McNicholl, reflect upon the PLE in prisons programmes operated by the Open University (through its Open Justice Centre). As well as providing insight into the benefit for law students the authors also explore the benefit for prisoners in the context of rehabilitative prison culture. The article considers why these opportunities have been offered to students, why the projects are designed as they are, what has been achieved and scope for future development. A variety of methods and programmes are explored here - the prison radio project and charity collaboration will, I'm sure, generate interest and ideas for those who work in this environment or are considering doing so.

Finally, Amy Wallace provides a review of Public Legal Education: The Role of Law Schools in Building a more Legally Literate Society authored by Richard Grimes and published by Routledge in May 2021. This thoughtful review will undoubtedly leave you wanting to read more and the information and resources provided within the book will prove useful to many of our readers. 